INVESTIGACIÓN/RESEARCH

Recibido: 03/03/2015---Aceptado: 07/04/2015---Publicado: 15/06/2015

\title{
Textos de la colección bicentenario: Nuestra historia republicana. Ciencias sociales de segundo año
}

Andrés Nasser Silva1: Universidad Metropolitana (Venezuela) anasser@correo.unimet.edu.ve

\section{RESUMEN}

El presente trabajo de investigación tiene por objeto el análisis, comparación y construcción de una crítica constructiva a los textos de la colección bicentenario implementados por el gobierno de Venezuela. Partiendo de la idea de que cada historiador reconstruye la historia basado en sus intereses y objetivos, es entonces que iniciaremos un recuento de lo que contiene el texto Nuestra historia republicana, específicamente lo relacionado al capítulo que trata el gobierno de Rómulo Betancourt y los primeros gobiernos de Rafael Caldera y Carlos Andrés Pérez. Ello por cuanto, los tres personajes resultarán emblemáticos para le historia republicana de Venezuela, ya sea por sus aportes o por sus decisiones políticas, sociales y económicas. Cada gobierno será reseñado según aparece en el texto de la colección bicentenario y luego será comparado con obras fundamentales de otros historiadores venezolanos. La comparación brindará la oportunidad de contrastar visiones y comprobar como la historia tendrá, usualmente, un importante sesgo pues la investigación suele tiene causas particulares.

\section{PALABRAS CLAVE}

Historia - Venezuela - bicentenario - Betancourt - Caldera - Pérez.

\section{Texts of the bicentennial collection: Our republican history. Second year Social Sciences}

\section{ABSTRACT}

The present work of research has as its aims the analysis, comparison and building of a constructive criticism of the texts of the bicentennial collection implemented by the government of Venezuela. Starting from the idea that each historian reconstructs

$1 \quad$ 1Andrés Nasser Silva: Universidad Metropolitana / Departamento de Estudios Jurídicos y Políticos / Caracas / Venezuela. anasser@correo.unimet.edu.ve 
the history based on his interests and objectives, then we begin a count of what the text Our republican history contains, specifically what is related to the chapter dealing with the government of Rómulo Betancourt and the first governments Rafael Caldera and Carlos Andrés Pérez. This because the three characters will become emblematic for the republican history of Venezuela, either for their contributions or their political, social and economic decisions. Each government will be reviewed as it appears in the text of the bicentennial collection and then it will be compared with other major works of Venezuelan historians. The comparison will provide an opportunity to compare views and verify how the story will usually have a significant bias as research often has particular causes.

\section{KEY WORDS}

History - Venezuela - bicentennial - Betancourt - Caldera - Perez.

\section{INTRODUCCIÓN}

La reconstrucción histórica moderna, e incluso contemporánea, suele ganarse más fácilmente enemigos y con ellos, amplias, constantes y fuertes críticas hacia los métodos de presentación, discusión y análisis de los eventos. Especialmente tedioso es investigar los hechos, contextos y decisiones en las que están involucradas presidentes o figuras públicas que se encuentren con vida al momento de presentar una investigación histórica. Las razones podrían recaer, tanto en la lupa y objetivos del historiador, como en el manejo historiográfico que promueva el estado; Y es que resultará lógico que quienes promuevan tal o cual investigación, tengan un fin, puesto que la vida histórica de la humanidad suele estar sometida al juicio crítico del historiador de turno.

La particularidad y fines de cada investigación no son una limitante para el recuento histórico, son más bien un complemento al saber de la humanidad y la cultura de un país, en este caso, la de Venezuela. Resultará cónsono pensar que tras dieciséis años de llegada de la Hugo Chávez y lo que la retórica gubernamental ha logrado, la reconstrucción histórica haya variado tendenciosamente hacia la lupa socialista, revolucionaria y porque no, comunista, del gobierno venezolano. No es una afirmación a la ligera, por cuanto más adelante en este ensayo se verá la tendencia oficial a defender las iniciativas del Partido Comunista venezolano y sus variables guerrilleras. Tampoco es una crítica, es el repaso de lo que significa la política oficial y su posición para con la historia republicana de Venezuela.

En el transcurso del ensayo abordaremos, por partes, dos capítulos relacionados a los gobiernos de Rómulo Betancourt, Raúl Leoni, Rafael Caldera y Carlos Andrés Pérez, vistos desde la presentación del texto Nuestra historia republicana. Allí expondremos sintetizadamente esos cuatro gobiernos, luego abordaremos esos mismos períodos presidenciales bajo la lupa de otros historiadores venezolanos, para finalmente concluir con el contraste y sentido de la historia en ambas expresiones: la del texto oficial y la de otros historiadores.

\section{OBJETIVOS}


- Exponer los gobiernos de Rómulo Betancourt, Raúl Leoni, Rafael Caldera y Carlos Andrés Pérez, según el libro Nuestra historia republicana.

- Presentar los gobiernos anteriores según otros investigadores.

- Contrastar ambas visiones de la historia venezolana partiendo de los objetivos anteriores.

\section{METODOLOGÍA}

A partir de diferentes fuentes especializadas se estudia, analiza y compara las diferentes situaciones históricas.

\section{DISCUSIÓN}

El recuento histórico, como diría Luis Villoro (s/f), sirve para conocer un sector de la realidad, sea la que fomenta el estado a través de su retórica oficial o a la que se dedica cada investigador en busca de satisfacer sus propios fines. Afirmará William Shakespeare que el pasado es el prólogo del libro de nuestras vidas y partiendo de allí, es que la humanidad se remite al pasado para darle al presente una razón para existir. Pero entonces cuando volvemos a Villoro (s/f), vemos como la historia responde a requerimientos del presente. En este punto nos detendremos unas cuantas líneas, y es que esa afirmación de Villoro (s/f) podría ser la punta de lanza de la versión oficial sobre la historia de Venezuela. Si con anterioridad la reconstrucción de nuestra vida política se centró en la consolidación de los proyectos, ideas y propuestas de los gobiernos anteriores al de Hugo Chávez, a partir del 2009 podríamos considerar que se ha empezado a adaptar la historia a los moldes del presente, en buena medida porque en Venezuela hay una tendencia a personalizar todo, desde las leyes hasta los nombres de los hospitales y por otra parte porque la ideología oficial precisa hacer un recuento de una parte de la historia que no se contó.

Luis Villoro (s/f) expondrá que "es frecuente que los intereses particulares del historiador, ligados a su situación, dirijan intencionadamente la selección de los datos, la argumentación y la interpretación, a modo de demostrar la existencia de una situación pasada que satisfaga esos intereses" (p41). Como se aprecia, no es menos cierto que la reconstrucción de la vida de un país sea el modelo dispuesto y propuesto por la ideología oficial, lo que no implica descartar o ignorar aquellas que no calzan en ese molde, pero si seleccionar los eventos y situaciones que planteen necesidades sociales o colectivas en las que participe uno $u$ otro grupo. La historia es un de las formas culturales de justificación institucional, de creencias y hasta de propósitos comunitarios. Hoy en día, como iremos desglosando más adelante, veremos como el texto Nuestra historia republicana responde a esos propósitos 
comunitarios que, inicialmente, atraparon a una colectividad que había sido olvidada por gobiernos anteriores al de Hugo Chávez.

Los capítulos que analizaremos en este ensayo, relativos a las presidencias de Rómulo Betancourt, Raúl Leoni, Rafael Caldera y Carlos Andrés Pérez de Nuestra historia republicana vendrán a corroborar, o no, lo que antes hemos introducido sobre como la ideología o retórica oficial es capaz de reconstruir la historia republicana del país, en función de sus intereses. Maurice Halbwachs (1968), afirmará que la historia no es todo el pasado y ciertamente no todos los sucesos individuales de un país deberían conformar su reconstrucción, pero la mayor parte de los hechos colectivos si, necesariamente, deben ser considerados como sustento para validación de la investigación histórica. Aun así, los acontecimientos pasados son elegidos, cotejados y clasificados siguiendo necesidades y reglas, lo que no implica que ciertos eventos del pasado como lo podrían ser, las torturas, las guerrillas y sus acciones y la mano dura de un gobierno, no puedan ser restablecidas. Es decir, si bien hay buena parte de la historia que podemos leer en libros y ensayos, hay otra que sólo es accesible gracias a un trabajo minucioso de los historiadores, memorias pequeñas o grandes que se creían perdidas (Halbwachs, 1968).

Veamos en este momento el primer capítulo que analizaremos del libro Nuestra historia republicana, dedicado a los gobiernos de Rómulo Betancourt (1958 - 1963) y Raúl Leoni (1964 - 1969). De entrada tenemos unas breves líneas exponiendo parte del discurso de juramentación de Rómulo Betancourt, en el que se realiza una breve crítica al Partido Comunista y como su ideología podría ser perjudicial para la construcción del estado democrático venezolano. Una de las principales críticas que recorre este capítulo es el relacionado al Pacto de Punto Fijo y su posición frente al comunismo y sus distintas variables en la vida política venezolana. Especialmente relevante resulta el hecho de lo que sucedió en el primer año de gobierno de Betancourt en la Organización de Estado Americanos cuando se discutían medidas contra el Gobierno cubano. Ignacio Luis Arcaya, para entonces canciller de Venezuela, se opuso a cualquier tipo de intervención contra La Habana, decisión contraria a la línea gubernamental de Betancourt, especialmente lo que se conocería con el tiempo La Doctrina Betancourt. Con esto, Unión Republicana Democrática, uno de los partidos oficiales, se retiró del gobierno y del Pacto de Punto Fijo que no era otra cosa que una serie de medidas mínimas de estricto cumplimiento para la consolidación de la democracia. Más adelante en el mismo capítulo, empieza a realizarse un recuento de cómo el gobierno de Betancourt estuvo influenciado por una creciente influencia de los Estados Unidos y el Pentágono, especialmente luego de ganar la presidencia norteamericana Jhon Fitzgerald Kennedy, en 1961. Kennedy habría afirmado en su discurso de campaña que trabajaría por "evitar la propagación del comunismo en América Latina" (p130). Partiendo de esa influencia y de la victoria de Kennedy, se dice que Venezuela rompió relaciones diplomáticas con la República de Cuba, apoyando la resolución de la OEA en la que se expulsaba a Cuba del organismo en 1962. Luego se habla de la relevancia de la autodeterminación de los pueblos consagrada en la carta fundacional de la Organización de las Naciones Unidas. 
El surgimiento de la violencia social y política en agosto de 1959, debido a la eliminación del Plan de Emergencia que estipulaba la entrega de material de construcción a los ciudadanos desempleados y campesinos que se habían movilizado a Caracas, generó una rápida respuesta de la Guardia Nacional. Hacia 1960, las invasiones a los latifundios de los campesinos sin tierra también fueron reprimidas por el mismo cuerpo de seguridad. Estudiantes y trabajadores sufrieron las mismas consecuencias por sus manifestaciones, cuando, por ejemplo, los empleados de la CANTV paralizaron la empresa, o cuando estudiantes y liceístas se enfrentaron a las fuerzas policiales. Todas estas acciones generaron la suspensión de garantías por parte del gobierno de Betancourt, pero eso no silenció las protestas. Sigue la narración del libro con una justificación a la creación de las guerrillas urbanas en marzo de 1961, dirigidas por el Movimiento de Izquierda Revolucionaria y el Partido Comunista. Las primeras acciones guerrilleras estuvieron marcadas por el ataque a una casa de cubanos anticastristas en 1961, quienes supuestamente estaban cooperando con los organismos de inteligencia venezolanos y estadounidenses. Más adelante, sería secuestrado un avión de Avensa por tres estudiantes y dos obreros, así como también se sucedió el robo de obras de arte en el Museo de Bellas Artes y por último se organizaría la lucha armada como estrategia de oposición a la democracia burguesa y antipopular del punto fijismo.

Del mismo modo como existió una lucha guerrillera, también hubo un levantamiento militar en 1962, conocido por El Carupanazo. Donde militares patriotas, en palabras del texto Nuestra historia republicana, se alzaron contra el gobierno pero fueron prontamente derrotados, perseguidos y enjuiciados por las fuerzas militares leales el gobierno. Por último, con el decreto 752, se ilegalizó al Partido Comunista y al MIR, que anteriormente habían decretado la lucha armada contra el gobierno.

En los últimos párrafos sobre el gobierno de Betancourt, se realiza un recuento de otras medidas relevantes que el ejecutivo implementó en el período constitucional que le tocaba, mencionaremos la totalidad: reducción del diez por ciento de los sueldos y salarios debido a la crisis petrolera, devaluación del bolívar, reajuste y restricción de las contrataciones colectivas, alineación con la política de contención de los movimientos de liberación nacional (guerrillas), promulgación de la Ley de Reforma Agraria y la sanción de una nueva Constitución con carácter representativo, creación de la Corporación Venezolana de Petróleo, creación de la Organización de Países Exportadores de Petróleo, inauguración de las ciudades de San Félix y Puerto Ordaz y creación de la Corporación Venezolana de Guayana.

\subsection{La presidencia de Raúl Leoni (1964 - 1959)}

Inicia la presidencia Leoni con la alianza llamada de "ancha base" en la que AD, URD y el Frente Nacional Democrático, conformarían las filas del gobierno nacional. El Partido Comunista, por su parte decidió iniciar la paz democrática, retomando con eso las formas legales de oposición al gobierno. En el texto Nuestra historia republicana, 
se dice que con el gobierno de Leoni continuó la represión y además surgió la figura del desaparecido, todo ello como parte de la "Operación Exterminio" dentro del plan de pacificación. Hubo muchos asesinatos, presos políticos, allanamientos y torturas, entre los cuales se destaca el de Alberto Lovera, ligado al PCV y a los frentes de liberación nacional. En 1966, se allanaría la Universidad Central de Venezuela, haciendo presos a setecientos estudiantes.

Las obras durante el gobierno de Leoni, expuestas por el texto que se analiza, son: la primera etapa del Complejo Hidroeléctrico de Guayana, el puente sobre el río Orinoco, la creación del Banco de los Trabajadores de Venezuela, se firmó el Acuerdo de Ginebra (para solucionar pacíficamente los problemas con Guyana) y se logra la entrada de Venezuela a la Asociación Latinoamericana de Libre Comercio.

\subsection{Primer Gobierno de Rafael Caldera (1969 - 1973)}

Una de las primeras medidas del gobierno de Rafael Caladera fue la pacificación a través de la eliminación de la DIGEPOL, por cuanto el país aún se encontraba convulsionado por la guerrilla, creando un nuevo organismo, la DISIP. También creó un comité de Pacificación presidido por el cardenal Quintero, como mediador entre el gobierno y las guerrillas. Más adelante, con la aceptación del PCV de la política de pacificación, solo quedaban algunos grupos guerrilleros en el país presididos por Carlos Betancourt, Gabriel Puerta y Fernando Soto Rojas. En concordancia con estas medidas de pacificación que inició Caldera, también se restablecieron relaciones diplomáticas con Cuba, se iniciaron con China y otros países socialistas de Europa del Este, además de mantenerlas con Chile desde que ganase Salvador Allende en 1970.

En lo referente a la política educativa del gobierno, expone el texto que los ciudadanos de a píe rechazaron la violación a la autonomía universitaria de la Universidad Central de Venezuela, por lo que se empezó a considerar la misma como una política educativa represiva, puesto que se arremetió contra los universitarios. Estos movimientos universitarios estuvieron influenciados por movimientos sociales del mundo entero, pero específicamente por la revolución cubana, las guerrillas latinoamericanas, entre otros. Otra sorprendente medida del gobierno de Caldera, en palabras del texto, fue el decreto 120 que establecía la Educación Diversificada, así como el proceso de bachillerización de las Escuelas Técnicas, provocando su desaparición.

Por último, otras medidas del gobierno de Caldera expuestas en Nuestra historia republicana fueron: revaluación del bolívar, aumento del precio del barril petrolero, nacionalización del gas y reversión petrolera, construcción del complejo petroquímico El Tablazo, la represa en el río Santo Domingo, así como interesantes avances en la construcción del complejo hidroeléctrico José Antonio Páez en la mencionada represa. 


\subsection{Gobierno de Carlos Andrés Pérez (1974 - 1979)}

El período de Pérez, expuesto en el libro es bastante corto y limitado en cuanto a las obras y proyectos gubernamentales. El capítulo se ancla en el aumento exorbitante de los precios petroleros que generaron la reforma a la Ley de Hidrocarburos, adelantando la reversión de la industria petrolera en común acuerdo con las concesionarias extranjeras. Esa reversión preveía el pago de indemnizaciones por una cifra cercana a los cinco mil millones de dólares que, de esperar a 1983, no se hubiesen tenido que pagar. Se creó PDVSA, que comenzaría a funcionar como un Estado dentro de un Estado puesto que no seguían ninguna política petrolera oficial, sino que la misma era la de las transnacionales petroleras. Por esta medida de reversión, el estado venezolano perdió alrededor de cuarenta por ciento de participación en la explotación petrolera, quedando en veinte por ciento para los próximos veinte años.

En este gobierno también hubo una fuerte política represiva contra los dirigentes de oposición, específicamente el Profesor Jorge Rodríguez.

\subsection{Gobiernos de Betancourt, Leoni, Caldera y Pérez en la lupa de otros investigadores.}

\subsubsection{Gobierno de Rómulo Betancourt (1958 - 1963)}

Alfredo Tarre Murzi (1992) en una biografía dedicada al que hoy en día se denomina el Padre de la Democracia venezolana, inicia esta presidencia de Betancourt exponiendo sobre la relevancia que tuvo a partir de 1959 una serie de protestas callejeras y obreras que venían dándose desde la Junta Provisional. Esto como prólogo de la reacción del Partido Comunista a su exclusión del Pacto de Punto de Fijo, más no de la posibilidad de postular y ser electos a cargos públicos como de hecho ocurrió con Fabricio Ojeda, electo diputado y posteriormente guerrillero.

Más adelante, se toca el tema del llamado Plan de Emergencia y los motivos de su eliminación. Betancourt expone que dicho plan había absorbido más de mil millones de bolívares "so pretexto de pagar una especia de seguro de desempleo", además de que existía en el mismo una enorme fuente de corrupción administrativa que no estaba resolviendo el tema de fondo: la desocupación obrera (Murzi, 1992). La eliminación del Plan de Emergencia, vino de la mano con la creación de nuevos empleos para tratar de ocupar a la mano obrera cesante. 
La faceta presidencial de Betancourt podría destacarse por dos aspectos fundamentales, según Manuel Caballero (2008), ellos son: como dirá Simón Sáez Mérida, que Betancourt era percibido realmente como Presidente de la República y como Comandante en Jefe de las Fuerzas Armadas venezolanas, siendo tal vez, el primer hombre en disponer de ambos cargos sin el menor resquicio de dudas. El segundo aspecto fundamental, no ajeno a la Doctrina Betancourt para la política exterior, era la política petrolera con la fundación de la Organización de Países Exportadores de Petróleo tuvo en sus manos "uno de los carteles más influyentes de la economía mundial" (p151).

Con la llegada de Betancourt al poder en 1958, no sólo se abría en Venezuela la posibilidad de instaurar un régimen democrático, sino también la su característica principal como lo es la libertad de expresión, los partidos políticos, elecciones libres, respeto por los derechos humanos y pulcritud administrativa. Dice Manuel Caballero (1998) que uno de los aspectos más resaltantes de la democracia que instaura Betancourt, es la ruptura con el paternalismo o el hecho de atar el poder con una persona, ello considerando que la constitución creada en 1961 rompía con todos esos paradigmas, además de que su impulsor no aceptó la postulación a un tercer período presidencial. Esencial para este proyecto político, era el Pacto de Punto Fijo mencionado en líneas anteriores, y es que entre sus objetivos, tres de los cuatro partidos políticos se proponían lo siguiente: prolongación de la tregua político para evitar rupturas, defensa de la constitucionalidad, derecho a gobernar conforme al resultado electoral, defensa de las autoridades constitucionales, gobierno de unidad nacional, programa mínimo común con el fin de afianzar la democracia como sistema, entre otras cosas. En palabras de Andrés Stambouli (2002), con el Pacto de Punto Fijo "se conformó un sistema político de conciliación y compromiso entre fuerzas sociales heterogéneas, encargados de mantener la cohesión social y asegurar la estabilidad de la forma política naciente" (p130). Es así como el compromiso fijado en este pacto, representará la solución a la crisis de gobernabilidad que vivía Venezuela para el momento, puesto que facilitará la cooperación. Por último, citado por Caballero (2008), Germán Carrera Damas dirá que la promulgación del Estatuto Electoral hará de Betancourt "un personaje histórico" (p149), puesto que incorpora a la vida política a la mayoría de los venezolanos que no eran ciudadanos: mujeres, jóvenes en edad militar y analfabetas; "esto último hacía sujetos políticos a quienes no estaban muy lejos de ser simples objetos: los mayoritarios campesinos” (p149).

\subsection{Gobierno de Raúl Leoni (1964 - 1969)}

Fernando Morgado Bossio (2012), hace un breve recuento de lo que fue el gobierno de Raúl Leoni en términos de proyectos, haremos un breve recuento por áreas. Por su parte, en lo que refiere a la política de pacificación, Leoni promulgo la Ley de Conmutación de Penas, logrando poner en libertad a más de 250 procesados por la insurrección armada guerrillera. 
Educación: Leoni promueve la construcción de 963 planteles de educación primaria y secundaria, aumentando en $80 \%$ la matrícula escolar, se crea el Instituto de Cultura y Bellas Artes y la Fundación del Niño.

Salud: se crean el Hospital General de Cumaná, Carupano, Maturín, Puerto cabello, Upata, San Cristobal, Cabimas, Valencia y el Universitario de Maracaibo, además de poner en funcionamiento varios acueductos a lo largo y ancho del territorio nacional. Junto a la Organización Mundial de la Salud, Leoni implementa programas de apoyo a parturientas, madres en lactancia y prevención de enfermedades venéreas.

Vialidad: construcción del Puente de Angostura sobre el rio Orinoco, Puente Internacional de Arauca, la autopista Valencia - Puerto Cabello, 2569 kilómetros de vías y pavimentación de 3000 kilómetros. Se inicia la construcción de la Cota Mil y la Avenida Libertador en Caracas.

Viviendas: se construyen alrededor de 154000 viviendas para las clases más bajas y clase media, además de 19000 en el sector rural, junto a lo cual se crea las Entidades de Ahorro y Prestamo para la adquisición de viviendas y mejoras en las mismas.

En lo que refiere al sector industrial, el gobierno de Leoni fue de los más importantes, puesto que la utilización de la bonanza petrolera, además de para modernizar el país, sirvió para instalar la industria pesada en Venezuela. Tal es el caso de Alcasa, Petroquímica en Moron y El Tablazo, Centrales Termoeléctricas de La Cabrera, Las morochas, La Fría y Punto Fijo, aumentando así la producción de energía eléctrica y la expansión del hierro, el acero y el aluminio en la zona de Guayana.

\subsection{Gobierno de Rafael Caldera (1969 - 1973)}

En la página web del Rafael Caldera (ver referencias), es posible apreciar los principales o más destacados logros de esta presidencia. Enunciaremos algunos de ellos que han sido corroborados dentro del presente proceso de investigación:

- Se logra la pacificación del país, con la incorporación de los grupos guerrilleros a la vida democrática. Murzi (1992), citando a Betancourt, dirá que "la legalización de los comunistas disidentes fue conveniente para atomizar al tradicional archipiélago marxista - leninista" (p421). Es decir, fue una estrategia del estado para calmar al país.

- Desarrollo de una política exterior de amistad, en la que se establecieron relaciones con Cuba, la Unión Soviética. También se fortaleció a la OPEP.

- Incorporación de los trabajadores del campo a la Ley del Trabajo.

- Logró la construcción de 100.000 casas en un año. 
- Logró la reversión petrolera y la nacionalización del gas, aumentando la participación fiscal a 34,63 Bs por barril petrolero.

- Construyó el Poliedro de Caracas, Parque Central, los tribunales de la República, comenzó el desarrollo del Metro de Caracas y construyó el Hospital Miguel Pérez Carreño.

- Creó el Instituto Nacional de Parques, el Aeropuerto de La Chinita, la segunda etapa del Guri, el Ateneo de Caracas y el Museo de Arte Contemporáneo en Caracas.

Es imperioso destacar que las obras mencionadas anteriormente son, únicamente, un resumen de su quinquenio, en ningún caso se pretende excluir o incluir obras para la conveniencia de esta investigación. Solamente tomamos sus obras más destacadas, expresadas por Andrés Caldera Pietri en la página web oficial del ex presidente.

\subsection{Gobierno de Carlos Andrés Pérez (1974 - 1979)}

Fernando Morgado Bossio (2012), en su columna semanal en el diario digital El Tiempo, expone por segmentos, parte de lo que fue la política nacional e internacional implementada por el primer gobierno de Carlos Andrés Pérez. Expondremos algunas de sus proyectos:

Economía: nacionalización de empresas del Estado, especialmente el petróleo y el hierro, para lo cual se crea Petroleos de Venezuela. Se crea el Fondo de Inversiones de Venezuela y Corpoindustria.

Educación: expansión de la matrícula escolar en todos los niveles, aunado a un aumento en los sueldos de profesores docentes y la construcción de numerosos edificios para liceos, escuelas y universidades. Se crea el reconocido programa de becas Gran Mariscal de Ayacucho para la juventud venezolana, la biblioteca Gran Mariscal para la producción de libros y textos de autores venezolanos y por último el Sistema Nacional de Orquestas Juveniles.

Vivienda: se crean alrededor de 350.000 viviendas, estimulando la construcción además de todos los materiales necesarios como cemento, cabillas, arena, piedra y piezas sanitarias.

\section{CONCLUSIÓN}

\subsection{Comparar y analizar brevemente las dos visiones expuestas anteriormente}


La introducción al presente trabajo podría, en cierto sentido, sonar parcializada o sugerente por cuanto se inicia la propuesta de investigación histórica como una que cumple los objetivos un historiador o investigador, ya sea por motivación personal o por solicitud expresa del gobierno de un país. Reconstruir el hecho histórico, no es tarea fácil, especialmente cuando analizas las diferentes corrientes historiográficas que existen y como cada una de ellas pone el acento en uno u otro evento. En el caso de la historia de Venezuela, queda evidenciado como el proceso de recuperar la vida política, social y económica del país tiende a tener el enfoque de la política oficial, pero ello no debería implicar sólo contar una versión de la historia. El gobierno de Betancourt y Murzi (1992) lo expone, enfrenta una fortísima lucha armada que incluso atenta contra su vida, pero ese hecho no debería ser el único relevante para el historiador, puesto que en un quinquenio se hizo más que enfrentar las guerrillas. Pero del punto de vista del libro Nuestra historia republicana, el gobierno de Betancourt estuvo enfocado en satisfacer los intereses norteamericanos, encarcelar y perseguir guerrilleros y enfrentarse a Cuba y a los regímenes comunistas del mundo. Carlos Aguirre Rojas (s/f) en el Antimanual del mal historiador, propone que la historia debe relatarse destacando los logros de los vencidos y de los vencedores, ello porque la historia es multicausal y la cultura de un país está determinada por todos aquellos eventos del pasado que influenciaron a sus generaciones. La persecución guerrillera es un hecho con el que vivió Venezuela por nueve años, pero la relevancia del pacto democrático que se firmó en 1958 es también fundamental para la estabilización y consolidación de un proceso democrático que construyó importantes obras de infraestructura a lo largo y ancho del territorio nacional. Por su parte, George Duby (s/f), también nos habla de las intenciones de la historia y esa afirmación está muy ligada al objetivo final de que esta investigación, puesto que dependiendo con la lupa que se analice un hecho histórico es que será posible comprender el porqué de tal o cual afirmación. Ahora, si bien es cierto que los objetivos del autor son fundamentales, no deja de ser cierto que aun así, la historia debería reescribirse bajo la posibilidad de obtener una verdad demostrable y con la rigurosidad necesaria para tal efecto. De todas formas, dice Duby (s/f), que la trascendencia de algunas historias, dependen de cuanto se identifique la sociedad con ellas. La pregunta para el caso venezolano sería, ¿con cuál de las historias modernas de Venezuela que hoy estudiamos, nos identificamos?

Michael Halbwachs (1968), justamente habla sobre la memoria colectiva y la memoria histórica y lo primero que dice es que no son la misma cosa. En la psique de una parte de nuestra sociedad, están guardados los recuerdos de la lucha armada de los sesenta, las guerrillas, las bonanzas que trajeron los gobiernos de Acción Democrática y COPEI pero también los largos períodos en los que el petróleo distrajo a nuestros gobernantes de servir y solucionar los mayores problemas de nuestro país. La historia debe ser un puente con el pasado, no para imitarlo pero si para verla en la gran pantalla y analizar lo que fuimos e hicimos y así comprender lo que somos. Halbwachs (1968) afirma que la historia es seleccionada y clasificada en los libros según necesidades, que podrían ser sociales o políticas. Hoy, al leer el libro Nuestra 
historia republicana nos damos cuenta cuales son los detalles que importan y cuáles son las microhistorias que, luego de dieciséis años le están enseñando a los niños y jóvenes venezolanos. No son historias falsas, es la versión de los vencidos de la que hablaba Aguirre Rojas (s/f), entonces la crítica fundamental, o más bien el llamado de atención, sería a rescatar lo bueno de ambas versiones y reconstruir un texto completo que guíe y posibilite a nuestra sociedad a escoger cual tendencia ideológica perseguir, a hilar fino en la reconstrucción de los hechos ya que de la comprensión de nuestro pasado es que entenderemos nuestro presente.

\section{REFERENCIAS}

\section{Libros completos:}

Arráiz Lucca, Eguren, Guédez, Meier, Paiva, Solano, Stambouli. (2000). Venezuela, balance del siglo XX. Epsilon Libros: Caracas.

Caballero, M. (1998). La crisis de la Venezuela contemporánea (1903 - 2002). Ediciones Alfadil: Caracas.

Caballero, M. (2008). Contra la abolición de la Historia. Ediciones Alfa: Caracas.

Stambouli, A. (2002). La política extraviada. Fundación para la cultura urbana: Caracas.

Tarre Murzi, A. (1984). Rómulo. Editores Vadell Hermanos: Caracas.

\section{Articulos en papel y electronicos:}

Aguirre Rojas, C. (2001). Antimanual del mal historiador. Obtained the 15th of november of 2014 and available in:

http://www.ares.unimet.edu.ve/humanidades/bpthu26/Apoyo.html

Duby, G. (s/f). Escribir la historia. Obtained the 15th of november of 2014 and available in: http://www.ares.unimet.edu.ve/humanidades/bpthu26/Apoyo.html

Halbwachs, M. (1968). Memoria colectiva y memoria histórica. Obtained the 15 of november of 2014 and available in:

http://www.ares.unimet.edu.ve/humanidades/bpthu26/Apoyo.html

Hobsbaw, E. (1998). Historia del Siglo XX. Obtained the 15th of november of 2014 and available in: http://www.ares.unimet.edu.ve/humanidades/bpthu26/Apoyo.html

Morgado Bossio, F. (2012). Presidencia de Raúl Leoni. Obtained the 17th of november of 2014 and available in:

http://www.eltiempo.com.ve/opinion/columnistas/presidencia-dr-raul-leoni$\underline{19641968 / 55694}$

Sin autor. (2014). Rafael Caldera. Obtained the 16th of november of 2014 and available in: http:// rafaelcaldera.com/index.php?route=information/faq\&topic=10_102 
Villoro, L. (2005). Historia, ipara qué? Obtained the 15th of november of 2014 and available in: http://www.ares.unimet.edu.ve/humanidades/bpthu26/Apoyo.html

Without author. (2010). Nace el expresidente que le dio la tranquilidad a Venezuela. Obtained the 16th of november of 2014 and available in:

http://www.noticias24.com/actualidad/243924/nace-el-expresidente-que-le-dio-latranquilidad-a-venezuela-raul-leoni 